\title{
The Src Inhibitor AZD0530 Prevents the Development of Osteolytic Bone Disease in Multiple Myeloma
}

Roy Heusschen, $\mathrm{PhD}^{1}$, Josephine Muller, $\mathrm{MSc}^{1}$, Marilène Binsfeld, $\mathrm{MSc}^{1}$, Erwan Plougonven, $\mathrm{PhD}^{5}$, Nadia Mahli ${ }^{1}$, Geert Carmeliet, $\mathrm{MD}, \mathrm{PhD}^{6}$, Angélique Léonard, $\mathrm{PhD}^{5}$, Martine Cohen-Solal, $\mathrm{MD}, \mathrm{PhD}^{4}$, Karin Vanderkerken, $\mathrm{PhD}^{3}$, Yves Beguin, $\mathrm{MD}, \mathrm{PhD}^{1,2}$, Eline Menu, $\mathrm{PhD}^{3}$, Jo Caers, $\mathrm{MD}, \mathrm{PhD}^{1,2}$

${ }^{1}$ Laboratory of Hematology, GIGA-Research, University of Liège, Liège, Belgium, ${ }^{2}$ Division of Hematology, Department of Medicine, University and CHU of Liège, Liège, Belgium, ${ }^{3}$ Myeloma Center Brussels, Department of Hematology and Immunology, Vrije Universiteit Brussel, Brussels, Belgium, ${ }^{4}$ Department of Rheumatology, Hôpital Lariboisière, INSERM-UMR 606, Université Paris Diderot, Paris, France, ${ }^{5}$ PEPs (Products, Environment, Processes), Department of Chemical Engineering, University of Liège, Liège, Belgium, ${ }^{6}$ Laboratory of Clinical and Experimental Endocrinology, Department of Clinical and Experimental Medicine, KU Leuven, Leuven, Belgium

Destructive bone lesions due to osteolytic bone disease are a major cause of morbidity and mortality in multiple myeloma patients, occurring in more than $80 \%$ of cases. Underlying osteolytic bone disease is an uncoupling of the bone remodeling process, with an increased activity of osteoclasts and a decreased activity of osteoblasts. Current strategies to treat osteolytic bone disease focus on anti-resorptive agents, which do not rebuild bone loss. Src kinase has been implicated in both osteoclast and osteoblast function. In this study, we assessed the effect of Src inhibition with AZD0530 (saracatinib, Astra Zeneca) on the development of multiple myeloma and its associated osteolytic bone disease.

We first determined Src family kinase expression in the multiple myeloma microenvironment and found that patient-derived myeloma cells express Src at low levels but disease stage does not correlate with Src expression levels. In accordance with the literature, Src mRNA expression was found to increase during osteoclast differentiation and decrease during osteoblast differentiation in publicly available microarray datasets. Next, we validated an inhibitory role of AZD0530 on osteoclast differentiation and function. At a pharmacological relevant concentration of $1 \mu \mathrm{M}$, AZD0530 inhibited the differentiation of RAW264.7 osteoclasts (Oc.N/FOV: 15.5+-1.6 treated vs. 53+-1.5 non-treated). AZD0530 treatment appeared to hamper efficient progenitor cell fusion and osteoclast polarization, reflected by a decrease of CTSK and DC-STAMP mRNA levels and a defective actin ring formation in treated cultures, which culminated in a complete inhibition of bone resorption. When assessing the effect of AZD0530 on osteoblast function we found that AZD0530 inhibits osteoblast differentiation, with a decreased expression of OSX and OCN, and alters osteoblast morphology.

In vivo, AZD0530 did not alter myeloma cell bone marrow infiltration in both the 5TGM.1 (37+-6.3\% AZD0530 treated vs. $25.2+-6.7 \%$ non-treated) and 5T2MM (26.1+-7.7\% vs. 29.1+-6.4\%) murine multiple myeloma models. However, bone health was significantly improved in both models following treatment with AZD0530. In the 5TGM.1 model multiple trabecular bone parameters were restored to levels observed in healthy control mice following AZD0530 treatment, including BV/TV (11.7+-0.3\% treated vs. 6.4+-0.3\% non-treated), Tb.N. $\left(2.5+-6 \times 10^{\wedge}-2 / \mathrm{mm}\right.$ vs. $\left.1.7+-9 \times 10^{\wedge}-2 / \mathrm{mm}\right)$ and Tb.Th $(46.2+-1 \mu \mathrm{m}$ vs. $37+-$ $0.8 \mu \mathrm{m})$. These results were confirmed in the $5 \mathrm{~T} 2 \mathrm{MM}$ model, which displays a more severe osteolytic bone disease. In addition, AZD0530 treatment resulted in an increase in cortical thickness (157.8+$0.8 \mu \mathrm{m}$ treated vs. $151.4+-0.7 \mu \mathrm{m}$ non-treated) and a decrease in the number and size of cortical lesions in 5TGM.1 mice. Finally, our findings were corroborated by histomorphometric analyses. 
In conclusion, we report a potent inhibitory effect of the Src inhibitor AZD0530 on the development of osteolytic bone disease in multiple myeloma. Our results indicate that AZD0530 exerts this effect via the modulation of both osteoclast and osteoblast function. These findings warrant further study of the feasibility and efficacy of AZD0530 to treat osteolytic bone disease in multiple myeloma patients. 